\title{
Glucose-lowering therapies and cancer risk: the trials and tribulations of trials and observations
}

\author{
J. A. Johnson • Y. Yasui
}

Received: 9 April 2010 /Accepted: 12 April 2010 /Published online: 4 June 2010

(C) Springer-Verlag 2010

Keywords Cancer · Controlled trials · Glitazones .

Malignancy $\cdot$ Metformin · Observational studies .

Type 2 diabetes

\section{Abbreviations \\ RECORD Rosiglitazone Evaluated for Cardiovascular Outcomes and Regulation of Glycaemia in Diabetes}

ADOPT A Diabetes Outcome Progression Trial

The potential role of glucose-lowering therapies in modulating the relationship between diabetes and cancer has seemingly exploded into our collective consciousness in the past year. While this relationship has been suggested for sometime [1-3], the recent surge in attention followed publication of several observational studies from European administrative registries and clinical databases [4-7]. This prompted a number of editorials and commentaries, most often critical of the observational nature of the reports, as well as critical of limitations in the analytic approach to those data [8-11]. Further, as is often the case with controversial reports from observational studies, calls were made to look to a higher level of evidence, such as data from randomised controlled trials.

To that end, a number of more recent publications have contributed data on this topic from randomised trials, including two meta-analyses of trials of long-acting insulin analogues $[12,13]$. Both of these concluded there was no

J. A. Johnson $(\bowtie) \cdot$ Y. Yasui

School of Public Health, University of Alberta,

Edmonton, AB, Canada T6G 2E3

e-mail: jeff.johnson@ualberta.ca increased risk of cancer, although the trials included involved small numbers of patients with type 1 and type 2 diabetes and were generally of very short duration, highlighting some of the major limitations of randomised trials in answering questions regarding potential modification of cancer risk. Other contributions have been the posthoc analysis of large randomised trials of glucose-lowering therapies, such as the paper by Home et al. in this issue of Diabetologia [14]. In that paper, the authors report on the risk of malignancies associated with the oral glucoselowering treatments to which patients with type 2 diabetes were randomised in the RECORD (Rosiglitazone Evaluated for Cardiovascular Outcomes and Regulation of Glycaemia in Diabetes) [15] and ADOPT (A Diabetes Outcome Progression Trial) [16] trials. These two trials were larger and of longer duration, overcoming some of the limitations of the trials in the meta-analysis of the insulin analogues. Nonetheless, the paper by Home et al. shares a common characteristic with these meta-analyses, in that they were all unplanned post hoc analyses from those trials. As such, it must be remembered that, while the data arose from randomised trials, they are still, after all, just observations.

Home and colleagues are careful to recognise this point in the discussion of their observations, where they indicate that the data merely suggest that, with respect to cancer risk, rosiglitazone offers no significant advantage over metformin. However, with regard to these two agents relative to sulfonylureas, the data are interpreted as being somewhat more suggestive of a potential benefit [14]. Furthermore, the authors point out that their findings are in keeping with previous observational studies of oral glucose-lowering therapies [1-3], including the relative reduction of cancer deaths observed in the UK Prospective Diabetes Study (UKPDS) metformin study [17]. Consis- 
tency, as Hill noted, is one of the important considerations when attempting to draw inferences of causality from epidemiological associations [18]. Under the view that data from randomised controlled trials provide higher level evidence, this consistency may be taken as reassurance that the previous observational studies were not insurmountably confounded.

While Home and colleagues report on post-hoc observational studies of two randomised trials, their strengths lie in the quality of the data, collected prospectively following the protocols for the original trials. One wonders, however, to what extent the trial protocols may have introduced potential biases, not fully addressed in these post-hoc observational studies of cancer risk. In the case of RECORD [15], the protocol had two therapy-associated strata, each with two randomisation arms. Thus patients on metformin monotherapy were randomised to sulfonylurea or rosiglitazone, and patients on sulfonylurea monotherapy were randomised to metformin or rosiglitazone. Patients were followed for a mean of 5.5 years, with a primary outcome of fatal or non-fatal cardiovascular events. Importantly, the RECORD protocol included a glycaemic control target of $\mathrm{HbA}_{1 \mathrm{c}} 7.0 \%$, to be achieved through dosage escalation of the randomised therapies, with rescue therapy consisting of addition of a third oral glucoselowering agent or transfer to insulin in patients not achieving a target $\mathrm{HbA}_{1 \mathrm{c}}$ of $8.5 \%$. Therein lies an important consideration that may not have been fully considered by Home and colleagues. While the analyses focused on the association between malignancies and the original randomised treatments, there is considerable evidence that the 'rescue' glucose-lowering therapies, added as part of the trial protocol, have known independent associations with cancer risk. It is also important to note that the initial treatments for patients coming into the RECORD trial (metformin or sulfonylurea monotherapy) were not randomly assigned. Failing to account for these additional exposures after randomisation over time and the nonrandomised nature of the initial therapy may result in cancer risk changes being falsely attributed to the randomised assignments of the second therapy in the RECORD trial.

A defence against this contention would be a view that, in conventional survival analysis, allocation to treatment groups and other covariates must be determined before follow-up starts. Pocock and Smeeth $[9,10]$ recently argued that, given a complex array of factors, many of which are unmeasured or unknown, it is usually impossible to accurately gauge the extent and even the direction of bias due to changes in drug therapy during follow-up. Strictly speaking, the effect of a time-varying drug therapy exposure is confounded by the condition that underlies the time-dependent change of that exposure, unless the time- varying covariate is changing randomly over time. In this case, however, applying the 'golden rule' for conventional survival analyses could actually lead to cancer risk differences being falsely attributed to the randomised allocation of treatment.

Under the RECORD protocol, randomisation would have ensured a balance between patients of the treatment arms. Thus, any differences in use of add-on rescue therapy between the arms were presumably attributable to the degree to which the randomised therapies controlled blood glucose. Add-on rescue therapy is therefore not independent of the original randomisation. As such, the originally randomised drug that appeared to be associated with increased (or decreased) cancer risk may have had no direct effect on cancer risk. Instead, the drug may not have achieved adequate glucose control, inducing the use of addon therapies that might independently have increased (or decreased) cancer risk. Some may see this as a classic case of 'confounding by indication', which is likely to occur in observational studies where the reasons for additional therapies are not measured or recorded. Fortunately, this is not the case in the protocol-driven data collection of a randomised trial such as RECORD; the protocol dictated the use of add-on therapy, which was fully measured and recorded. Furthermore, it is highly unlikely, albeit unverifiable, that the reasons for add-on therapy were related to cancer risk, making confounding by indication improbable. In this view, accounting for the addition of rescue therapies known to be independently associated with cancer risk, perhaps with exposure definitions that recognise the timevarying nature of these exposures, could provide a better estimate of the risk associated with the randomised therapies. At the least, such a secondary analysis would be helpful to assess the consistency with the primary analysis results.

In fact, if we were to look to randomised trial data to inform the debate on specific glucose-lowering therapies and cancer risk, we find a similar situation for all recent large randomised controlled trials of glucose-lowering therapies for type 2 diabetes, given the particular focus on overall glycaemic control. For example, in the Prospective Pioglitazone Clinical Trial in Macrovascular Events (PROActive) Study [19], a large trial which randomised patients with type 2 diabetes to pioglitazone or placebo, differences in the rates of cancer as a secondary outcome were noted, with more cases of bladder cancer in the pioglitazone group and more breast cancer with placebo, but no difference overall in the incidence of cancer [20,21]. More importantly, while PROActive was a randomised trial of pioglitazone versus placebo, the investigators also aimed for participants to achieve $\mathrm{HbA}_{1 \mathrm{c}}$ below the recommended target, which they took to be $<6.5 \%$ [18]. Therefore, substantial changes to non-randomised medications 
occurred, specifically to other glucose-lowering agents. By the end of the study, the placebo group was using more metformin and more insulin than the pioglitazone group, while both groups had a similar decline in the use of sulfonylureas [19]. In the end, the pioglitazone group achieved a significant and clinically important difference in $\mathrm{HbA}_{1 \mathrm{c}}$, but no difference in the main primary outcome of mortality or cardiovascular events.

In RECORD, the rosiglitazone group also achieved a significantly greater reduction in $\mathrm{HbA}_{1 \mathrm{c}}$, but no differences were observed in the main outcomes of fatal or non-fatal cardiovascular events [17]. At best, secondary analyses of these large randomised controlled trials may be limited to assessing the relationship between improved cancer risk and overall glycaemic control, through whatever combinations of specific therapies used. With this in mind, these observations suggest that cancer risk is not reduced by improving glycaemic control in type 2 diabetes [22].

In considering the entire trajectory of glucose-lowering therapy in RECORD, Home and colleagues indicate that the use of rescue therapy and addition of insulin therapy were minimal, with the majority of follow-up time on the rosiglitazone [14]. However, recent revelations suggest that this may not have been the case, with $40 \%$ of patients no longer taking the drug by the end of the study [23]. While this newly revealed information calls into question the overall integrity of the RECORD trial, it also highlights the need to adequately record and measure exposure to glucose-lowering therapies in the analysis of secondary outcomes such as cancer.

Understanding the relationship between diabetes and cancer is perhaps one of the next biggest challenges for the clinical community. Clearly this also means a better understanding of the role of glucose-lowering therapies. Home and colleagues' observations from the RECORD and ADOPT trials affirm that a reasonably strong and consistent story is emerging as far as the association between metformin use and reduced cancer risk is concerned. Our current understanding is likely to be enhanced within the next few years, with data from ongoing randomised trials designed to answer the question of potential benefits of metformin and glitazones in the treatment or prevention of cancer [24]. Randomised controlled trials, whether in oncology or diabetes, are not the best sources of evidence of rare adverse events $[25,26]$. As such, we must continue to look to observational studies to inform this debate, seeking and sharing those with the highest quality data and analytic approaches that attempt to disentangle these complex exposure and outcome relationships.

Acknowledgements J. Johnson holds a Canada Research Chair in Diabetes Health Outcomes. Y. Yasui holds a Canada Research Chair in Biostatistics. Both authors are Senior Scholars with Alberta InnovatesHealth Solutions.
Duality of interest The authors declare that there is no duality of interest associated with this manuscript.

\section{References}

1. Yang YX, Hennessy S, Lewis JD (2004) Insulin therapy and colorectal cancer risk among type 2 diabetes mellitus patients. Gastroenterology 127:1044-1050

2. Evans JMM, Donnelly LA, Emslie-Smith AM, Alessi DR, Morris $\mathrm{AD}$ (2005) Metformin and reduced risk of cancer in diabetic patients. BMJ 330:1304-1305

3. Bowker SL, Majumdar SR, Veugelers P, Johnson JA (2006) Increased cancer-related mortality for patients with type 2 diabetes who use sulfonylureas or insulin. Diab Care 29:254-258

4. Hemkens LG, Grouven U, Bender R et al (2009) Risk of malignancies in patients with diabetes treated with human insulin or insulin analogues: a cohort study. Diabetologia 52:1732-1744

5. Colhoun HM, SDRN Epidemiology Group (2009) Use of insulin glargine and cancer incidence in Scotland: a study from the Scottish Diabetes Research Network Epidemiology Group. Diabetologia 52:1755-1765, Erratum Diabetologia 52: 2469

6. Jonasson JM, Ljung R, Talbäck M, Haglund B, Gudbjörnsdòttir S, Steineck G (2009) Insulin glargine use and short-term incidence of malignancies - a population-based follow-up study in Sweden. Diabetologia 52:1745-1754

7. Currie CJ, Poole CD, Gale EAM (2009) The influence of glucoselowering therapies on cancer risk in type 2 diabetes. Diabetologia 52:1766-1777

8. Garg SK, Hirsch IB, Skyler JS (2009) Insulin glargine and canceran unsubstantiated allegation. Diab Technol Ther 29:473-476

9. Pocock SJ, Smeeth L (2009) Insulin glargine and malignancy: an unwarranted alarm. Lancet 374:511-513

10. Smeeth L, Pocock SJ (2009) Authors reply. Lancet 374:1744

11. Duncan BB, Schmidt MI (2009) Metformin, cancer, alphabet soup and the role of epidemiology in etiologic research. Diab Care 32:1748-1750

12. Dejgaard A, Lynggaard H, Råstam J, Krogsgaard Thomsen M (2009) No evidence of increased risk of malignancies in patients with diabetes treated with insulin detemir: a meta-analysis. Diabetologia 52:2507-2512

13. Home PD, Lagarenne P (2009) Combined randomised controlled trial experience of malignancies in studies using insulin glargine. Diabetologia 52:2499-2506

14. Home PD, Kahn SE, Jones NP, Noronha D, Beck-Nielsen H, Viberti G, for the ADOPT Study Group and the RECORD Steering Committee (2010) Experience of malignancies with oral glucose-lowering drugs in the randomised controlled ADOPT and RECORD clinical trials. Diabetologia (in press). doi:10.1007/ s00125-010-1804-y

15. RECORD Study Team (2009) Rosiglitazone evaluated for cardiovascular outcomes in oral agent combination therapy for type 2 diabetes (RECORD): a multicentre, randomised, open-label trial. Lancet 373:2125-2135

16. Kahn SE, Haffner SM, Heise MA, Herman WH, Holman RR, Jones NP, Kravitz BG, Lachin JM, O’Neill MC, Zinman B, Viberti G, ADOPT Study Group (2006) Glycemic durability of rosiglitazone, metformin, or glyburide monotherapy. $\mathrm{N}$ Engl $\mathrm{J}$ Med 355(23):2427-2443

17. UK Prospective Diabetes Group (1998) Effect of intensive bloodglucose control with metformin on complications in overweight patients with type 2 diabetes. UKPDS 34. Lancet 352:854-865

18. Hill AB (1965) The environment and disease: association or causation? Proc R Soc Med 58:295-300

19. PROActive investigators (2005) Secondary prevention of macrovascular events in patients with type 2 diabetes in the 
PROActive Study (PROspective pioglitAzone Clinical Trial In macroVascular Events): a randomized controlled trial. Lancet $366: 1279-1289$

20. Goldstein MR (2006) PROActive Study. Lancet 367:23

21. Dormandy JA, on behalf of PROActive Writing Committee (2006) PROActive Study-authors' reply. Lancet 367:26-27

22. Gerstein HC (2010) Does insulin therapy promote, reduce, or have a neutral effect on cancers? JAMA 303:446-447
23. Nissen SE (2010) Setting the RECORD straight. JAMA 303:1194-1195

24. Clinical Trials Registry Website. Available from www.clinical trials.gov/ct2/search, accessed 30 March 2010

25. Laupacis A, Mamdani M (2004) Observational studies of treatment effectiveness: some cautions. Ann Intern Med 140:923-924

26. Papanikolaou PN, Christidi GD, Ioannidis JP (2006) Comparison of evidence on harms of medical interventions in randomized and nonrandomized studies. CMAJ 174:635-641 